\title{
The oxygen isotope effect in the earliest processed solids in the solar system: is it a chemical mass-independent process?
}

\author{
A. Ali and J. A. Nuth
}

\author{
Astrochemistry Laboratory, Solar System Exploration Division, NASA's Goddard Space Flight Center, Greenbelt, \\ Maryland 20771, USA \\ e-mail: Ashraf.Ali@gsfc.nasa.gov
}

Received 12 December 2006 / Accepted 20 March 2007

\begin{abstract}
Aims. An anomalous effect in the abundances of oxygen isotopes in the most refractory calcium-aluminum-rich inclusions (CAIs) was discovered some thirty years ago. The origin of these oxygen isotopic anomalies has hitherto remained unexplained. The origin is neither nuclear, nor has the recent photochemical self-shielding explanation been proven to be valid. We discuss a possible chemical mechanism to resolve these observed effects.

Methods. By uniting the most recent laboratory observations of nanoclusters of silicates in beams and the first principles theoretical studies of their structure and properties with a major dynamical constraint recently described as the surface non-RRKM effect during $\mathrm{SiO}_{2}$ formation on the growing grain, we show that the origin of the anomalous isotopic effect in high-temperature minerals in CAIs is chemical and strictly mass-independent.

Results. We report that the surface non-RRKM effect would represent a major process in the formation of our own solar system and observable protoplanetary accretion disks, and the mass-independent isotope effects are directly associated with the formation of primary grains in the high temperature nebular environment. We expect that this chemical reaction mechanistic approach combined with future time-resolved studies on the kinetics of growth of silicates and a precise knowledge of the oxygen isotopic abundances of the sun would provide a very detailed understanding of the origins of formation of our solar system.
\end{abstract}

Key words. astrochemistry - solar system: formation - stars: planetary systems: protoplanetary disks - molecular processes - Sun: abundances - ISM: evolution

\section{Introduction}

Based on radiometric age determinations (Allegre 2001), researchers have long argued that calcium-aluminum rich inclusions (CAIs) are the oldest known minerals that were formed by condensation reactions (Ali 1998) from a hot gas in the solar nebula. The separated minerals from these inclusions are numerous and include forsterite $(\mathrm{MgO})_{2} \mathrm{SiO}_{2}$, alumina $(\mathrm{AlO})\left(\mathrm{AlO}_{2}\right)$, spinel $(\mathrm{MgO})\left(\mathrm{Al}_{2} \mathrm{O}_{3}\right)$, anorthite $(\mathrm{CaO})\left(\mathrm{Al}_{2} \mathrm{O}_{3}\right)\left(\mathrm{SiO}_{2}\right)_{2}$ and melilite $(\mathrm{CaO})_{2}\left(\mathrm{Al}_{2} \mathrm{O}_{3}\right)\left(\mathrm{SiO}_{2}\right)$. Note that the chemical notation used here is different from the mineralogists' conventional formulas.

On the grounds of conventional wisdom in chemical thermodynamics (Urey 1947; Weston 1999), in a three-isotope plot the slope of $\left[\left({ }^{17} \mathrm{O} /{ }^{16} \mathrm{O}\right)_{\text {sample }} /\left({ }^{17} \mathrm{O} /{ }^{16} \mathrm{O}\right)_{\text {standard }}-1\right]$ versus the corresponding quantity for ${ }^{18} \mathrm{O}$ should be very close to 0.52 for processes that are strictly mass-dependent (Weston 1999). The first exception (Clayton et al. 1973) to this rule was found by Clayton and co-workers some thirty years ago. They observed a slope close to 1.0 , instead of 0.52 , in the oxygen in separated minerals from CAIs. The origin of this phenomenon of the covariation of ${ }^{17} \mathrm{O} /{ }^{16} \mathrm{O}$ and ${ }^{18} \mathrm{O} /{ }^{16} \mathrm{O}$ isotopic ratios with an equal magnitude of fractionation has remained a vexing problem since that time (Clayton et al. 1973). Based upon the premise (Urey 1947) that chemical processes would invariably produce mass-dependent oxygen isotopic compositions in the formation and growth of minerals, many scenarios (Clayton et al. 1973) have been invented in models of mixing of multiple (or at least two) isotopically different reservoirs in order to account the "slope 1" oxygen isotopic distributions of the oldest mineralogical components in CAIs. Once this oxygen isotopic effect was attributed by Clayton et al. (1973) to nucleosynthetic processes in stars, and they argued that the effect results from an admixture of a component of almost pure ${ }^{16} \mathrm{O}$-rich solids with the so-called normal solar values. There is nearly a complete lack of detection of "pure" enriched ${ }^{16} \mathrm{O}$ grains in meteorites (Nittler 2003). Clayton et al. (1973) also argued that the presence of excesses of ${ }^{24} \mathrm{Mg}$ and ${ }^{28} \mathrm{Si}$ in those samples in which the ${ }^{16} \mathrm{O}$ excess has been found should be detectable. After more than thirty years, no such correlated isotopic abundances have been identified. It is ironic that these observations now certainly argues against nuclear explanation that was advanced earlier. The photochemical self-shielding (Clayton 2002; Lyons \& Young 2005; Yurimoto \& Kuramoto 2004) effect in CO under different astrophysical settings has been hypothesized as an alternative mechanism for production of the observed anomalies. The transfer of anomalous oxygen from the isotopically selective photolysis of $\mathrm{CO}$ to grains has been modeled for nebular environments. Thiemens (2006) has critically reviewed that the recent self-shielding explanations are not applicable to the origin of oxygen isotope anomalies in solids and especially in silicates in the early solar nebula. For the sake of brevity, we would present only three key arguments against self-shielding effect (Thiemens 2006). The rapid isotopic exchange reactions under nebular conditions would put a major limit on sequestering anomalous oxygen isotopes that may be produced by the optical shielding effect. Second, in all 
models of CO self-shielding, the major problem lies in the actual process of photochemistry. The self shielding process occurs in the spectral region between 90 and $110 \mathrm{~nm}$, and it is assumed that for the $\mathrm{CO}$ there exist discrete isotopic absorption lines. Because of the non adiabatic coupling (Andric et al. 2004) between discrete states and the dissociative continuum state in $\mathrm{CO}$, most of the absorption profiles are wider and a considerable overlap occurs. All self-shielding models have not accounted this important (Thiemens 2006; Andric et al. 2004) lack of discrete isotopic states, and in reality there may not be any self-shielding in CO. Third, in one scenario (Yurimoto 2004) of photochemical self-shielding models, it was proposed that the origin of oxygen isotope heterogeneity is the parent molecular cloud. The anomalous oxygen is trapped in icy dust grains and is transported to the inner solar nebula. The model suffers from two problems: (1) our poorest understanding in astrochemistry is the gas-dust interaction problem, and formation of ice on silicate grains in dense molecular clouds; (2) it is not understood that if the anomalous oxygen isotopes are at all participating in reactions on grain surfaces at low temperatures and trapped in ice, how does the isotopic composition get transferred to the silicate solid in the inner disk of proto-sun? At present this model of molecular cloud origin is very weak.

\section{Surface reactions in high-temperature clouds}

Here we would like to demonstrate the mass-independent gas phase (Hathorn \& Marcus 1999, 2000; Gao \& Marcus 2002, 2001; Charlo \& Clary 2004) and surface reactions (Marcus 2004) in the formation and growth of solids in protoplanetary accretion disks as a possible chemical mechanism for the observed oxygen isotopic effects (Clayton et al. 1973) in mineral separates in CAIs. We will present three key arguments - based on recent laboratory experimental findings (Reber et al. 2006) of the compositional distributions of cluster intermediates during nucleation of $\mathrm{SiO}$, the first principles density functional theoretical interpretation (Reber et al. 2006) of their growth mechanisms, and astronomical observations of silicate formation in planet-forming circumstellar disks (van Boekel et al. 2004). What follows will show that the "slope1" oxygen isotopic distributions are due to non-RRKM effect in unimolecular steps in bimolecular reactions during $\mathrm{SiO}_{2}$ formation on the surface of a growing cluster.

Table 1 represents the relative intensities of the clusters $(\mathrm{SiO})_{n}\left(\mathrm{SiO}_{2}\right)_{m}$ shown in Fig. 1 in Reber et al. (2006). These clusters form from $\mathrm{SiO}$ molecules laser-vaporized from $\mathrm{SiO}$ solid under a helium atmosphere. The clusters $(\mathrm{SiO})_{n}$ containing 2, 3 , and 7 units have higher intensities than other sizes. There is a substantial decrease in intensity in going from $n=3$ to $n=4$, and there is also a marked increase in intensity in going from $n=6$ to $n=7$. The experimental observation of intensity distributions of clusters $(\mathrm{SiO})_{n}$ is consistent with the calculated (Reber et al. 2006) intrinsic binding stability of their ground state structures. The gradient corrected density functional theoretical studies (Reber et al. 2006) on the geometry, electronic structure and stability of clusters suggest that $(\mathrm{SiO})_{n}$ clusters containing up to 4 units are single rings. The $\mathrm{Si}-\mathrm{Si}$ bond appears first at $\mathrm{Si}_{5} \mathrm{O}_{5}$, and starting at this size, the elementary rings begin to assemble into multiple rings that eventually lead to cages. It was shown that the most stable ground state structures at larger sizes have a central core of pure $\mathrm{Si}$ atoms surrounded by more oxygen-rich and particularly $\mathrm{SiO}_{2}$ stoichiometry, outer shells. These $(\mathrm{SiO})_{n}$ clusters have many chemical isomers and the number of isomers increases as the cluster size increases. To reach the energetically most stable configuration of a cluster of a given size, atomic oxygen must be transported from the core to the interface of silicon atoms in Fig. 2 in reference (Reber et al. 2006). We refer the oxygen atoms on the Si-rich surface as adsorbates or $\mathrm{O}(\mathrm{ads})$ in the discussion that follows. As cluster growth occurs by the addition of the $\mathrm{SiO}$ molecule, the reaction between an atomic oxygen and silicon monoxide molecule on the surface of a cluster is of interest, as the cluster itself isomerizes to the most stable ground state geometry. We argue in this brief communication that the experimental observation of gradual oxygen enrichment (Table 1) upon cluster growth and predictions (Reber et al. 2006) of their structures as a core of $\mathrm{Si}$ atoms surrounded by a shell of $\mathrm{SiO}_{2}$ units molecularly bonded to the surface of the core, have profound implications for the theory (Marcus 2004) of chemical mass-independent oxygen isotopic effects during the formation of the earliest silicates (van Boekel et al. 2004) in the solar system.

The ratio of oxygen to silicon in $\mathrm{SiO}$ is 1 while it is 2 for $\mathrm{SiO}_{2}$. One of the important questions is whether this oxygen enrichment occurs in a single step or through the gradually evolving stoichiometry of the growing cluster during the reactive $\mathrm{SiO}$ growth processes. The second row in Table 1 represents relative intensities of the $\mathrm{Si}_{n} \mathrm{O}_{n+1}$ clusters that contain only one additional oxygen atom. The agreement between the observed intensity distributions of these clusters and the calculated (Reber et al. 2006) energetics of bimolecular reactions based on the properties of clusters led us to propose the microscopic pathways of their formation in the beam. It was shown that $\mathrm{Si}_{5} \mathrm{O}_{5}$ is the smallest cluster with the $\mathrm{Si}-\mathrm{Si}$ bond to start the oxygen enrichment process wherein the generated $\mathrm{Si}_{5} \mathrm{O}_{6}$ combines with selected $(\mathrm{SiO})_{n}$ clusters to form fragments that are even richer in oxygen. So an enriching cascade involves $\mathrm{Si}_{5} \mathrm{O}_{6}, \mathrm{Si}_{4} \mathrm{O}_{5}, \mathrm{Si}_{3} \mathrm{O}_{4}$, $\mathrm{Si}_{2} \mathrm{O}_{3}$, as the intermediate products and $\mathrm{SiO}_{2}$ as the terminal member in the sequence of reactive processes during condensation. This experimental observation along with a reasonable theoretical interpretation emphasizes that the molecule $\mathrm{SiO}_{2}$ is not the precursor species and does not play any direct role in the gas phase in the formation of cluster intermediates (Table 1) and their growth and evolution to bulk silicates. Thus the most fundamental assumption (Thiemens 1996) that the oxygen isotopic anomalies in CAIs and chondrules (solid silicon oxide condensates) are symmetry derived from initial gas phase reactions involving $\mathrm{O}+\mathrm{SiO} \rightarrow \mathrm{SiO}_{2}$ or $\mathrm{OH}+\mathrm{SiO} \rightarrow \mathrm{SiO}_{2}+\mathrm{H}$ turns out to be invalid based on these experiments.

The laboratory experiment (Reber et al. 2006) also shows the presence of an additional number of oxygen atoms in the observed distribution of $\mathrm{Si}_{n} \mathrm{O}_{m}$ clusters. Such cluster distributions can be decomposed into multiples of $(\mathrm{SiO})_{n}$ and $\left(\mathrm{SiO}_{2}\right)_{m}$ units (Table 1). The relative intensities of these clusters are also displayed in Table 1 . The appearance of these clusters in the mass spectra (Reber et al. 2006) indicates that an oxidation process is occurring. The oxidation in the solar nebula could occur by reactions with $\mathrm{H}_{2} \mathrm{O}$ molecules, or with free oxygen atoms in more oxidative environments close to the protostar in accretion disks. In the experiment, the oxidation is most likely due to the collision of a cluster with the dimer $\mathrm{Si}_{2} \mathrm{O}_{2}$. The collision process eventually could leave an oxygen atom on the surface of the cluster and form the $\mathrm{Si}_{2} \mathrm{O}$ molecule in the gas phase, the dominance of which is clearly seen in the mass spectra (Reber et al. 2006). In order to understand the oxidation of a cluster at high temperatures one must account for the effects of both diffusive transport and surface heterogeneous chemistry. At high temperatures, a $\mathrm{SiO}$ molecule would be transported from the interior core of the growing cluster to the surface and the reaction between the $\mathrm{O}$ atom and the $\mathrm{SiO}$ molecule would therefore start on the 
Table 1. Relative intensities of the clusters $(\mathrm{SiO})_{n}\left(\mathrm{SiO}_{2}\right)_{m}$. Intensities displayed reflect the relative abundances of cluster cations observed (Reber et al. 2006) in time-of-flight mass spectra following laser ionization of neutral species formed by condensation reactions of $\mathrm{SiO}$ molecules. Because of the use of a femtosecond laser and therefore a nearly fragmentation free multiphoton ionization process, it is assumed within the limitations of the current experiment that the observed distributions represent the nascent distribution of neutral clusters.

\begin{tabular}{ccccccccc}
\hline \hline & $n=1$ & $n=2$ & $n=3$ & $n=4$ & $n=5$ & $n=6$ & $n=7$ & $n=8$ \\
\hline$m=0$ & 685.2 & 655.4 & 255.1 & 71.3 & 58.1 & 36.4 & 89.3 & 38.1 \\
$m=1$ & 99.0 & 99.6 & 57.2 & 50.9 & 47.8 & 34.9 & & \\
$m=2$ & 49.9 & 48.2 & 51.4 & 35.9 & 33.6 & & & \\
$m=3$ & 49.2 & 47.0 & - & 36.7 & & & & \\
\hline
\end{tabular}

surface. Such a surface mediated process would not only result in the observation of clusters of the form $(\mathrm{SiO})_{n-1}\left(\mathrm{SiO}_{2}\right)_{m+1}$ but also of the form $(\mathrm{SiO})_{n-1}\left(\mathrm{SiO}_{2}\right)_{m}$ due to the evaporative loss of those $\mathrm{SiO}_{2}$ molecules that are long lived (Marcus 2004) on the surface. The experiment clearly shows the surface oxidation chemistry and the resulting unique cluster distribution (Table 1) that we observe.

In a pioneering theoretical paper, Marcus (2004) recently proposed that the mass-independent oxygen isotope effect in CAIs occurs during the formation of $\mathrm{SiO}_{2}$ on the surface of a growing mineral grain. In a series of published papers Marcus and his co-workers (Harthorn \& Marcus 1999, 2000; Gao \& Marcus 2002, 2001) for the first time explained that the unusual oxygen isotopic effect in stratospheric ozone is a matter of understanding the preferential collisional stabilization of the asymmetric isotopomers relative to the ones that are symmetric. A non-statistical (non-RRKM) correction, referred to as an $\eta$ effect (Gao \& Marcus 2001), was introduced for the density of the quantum states of the vibrationally excited ozone owing to the lack of redistribution of the energy among its vibrationalrotational modes during the typical lifetime of the activated molecule. The correction was larger for the symmetric isotopologue than for the asymmetric one. Marcus's theory (2004) pointed out that this gas phase, non-RRKM explanation is not applicable to chemical mass-independent fractionation effects during the formation of silicates in solar nebular environments. Although the volume-based $\eta$ effect (Gao \& Marcus 2001) could occur for the gas phase $\mathrm{SiO}_{2}$ molecule, the experiments reveal that the $\mathrm{SiO}_{2}$ molecule does not play any direct role in the gas phase in the origin of silicate grains in circumstellar environments. Instead (Marcus 2004), silicate formation involves the surface analogue of the volume-based $\eta$ effect for ozone with reactions on the surface, as in

$\mathrm{SiO}($ ads $)+\mathrm{O}($ ads $) \rightleftharpoons \mathrm{SiO}_{2}^{*}($ ads $) \rightarrow \mathrm{SiO}_{2}(\mathrm{~g})$.

We emphasize recent analysis of the laboratory and observational data that suggest that $\mathrm{O}$ (ads) would primarily form during the vaporization and re-condensation cycle in protoplanetary accretion disks from either (Marcus 2004) $\mathrm{H}_{2} \mathrm{O}+$ Surface $\rightarrow$ $\mathrm{H}_{2}+\mathrm{O}$ (ads) or (Reber et al. 2006) $\mathrm{Si}_{2} \mathrm{O}_{2}(\mathrm{~g})+$ Surface $\rightarrow$ $\mathrm{Si}_{2} \mathrm{O}(\mathrm{g})+\mathrm{O}(\mathrm{ads})$ or $\mathrm{O}$ migration to a $\mathrm{Si}$ in the core of an $\mathrm{Si}_{x} \mathrm{O}_{y}$ cluster (Reber et al. 2006). If a surface non-RRKM effect (Marcus 2004) exists, this would yield $\mathrm{SiO}_{2}^{*}$ (ads) that is mass-independently rich in ${ }^{17} \mathrm{O}$ and ${ }^{18} \mathrm{O}$, and $\mathrm{SiO}$ (ads) + O (ads) that is mass-independently poor in the two heavier oxygen isotopes. The $\mathrm{SiO}_{2}^{*}$ (ads) is deactivated by vibrational energy loss to the cluster and leaves the surface, whereas the other species, $\mathrm{O}$ (ads) and $\mathrm{SiO}$ (ads) are incorporated into the growing cluster surface to form silicates. In the region of protoplanetary accretion disks where CAIs form, temperatures are of the order of 1500 to $2000 \mathrm{~K}$. Because asymmetric $\mathrm{SiO}_{2}^{*}$ (ads) have relatively longer lifetimes compared to the symmetric species, the evaporation process for dynamical reasons would fractionate oxygen isotopes mass-independently at high temperatures either by the loss of vibrational energy to the growing grain or by the attack of a reductant, even if the process is endothermic. In the light of heterogeneous catalytic surface chemistry, large clusters such as $(\mathrm{SiO})_{n}\left(\mathrm{SiO}_{2}\right)_{m}$ shown in Table 1 , as they grow would be enriched in ${ }^{16} \mathrm{O}$ and depleted in ${ }^{17} \mathrm{O}$ and ${ }^{18} \mathrm{O}$ by equal amounts. The relative intensities of the clusters $(\mathrm{SiO})_{n}\left(\mathrm{SiO}_{2}\right)_{m}$ displayed in Table 1 reflect that the surface non-RRKM effect may indeed exist, otherwise we would not have observed the chemically anomalous $\mathrm{Si}_{n} \mathrm{O}_{m}$ compositions in the experiment. The most important aspect of this observation of anomalous $\mathrm{Si}_{n} \mathrm{O}_{m}$ clusters is the formation of transient $\mathrm{SiO}_{2}$ on the surface of cluster.

\section{Chemical pathway (microscopic) description of the growth process of high temperature refractory silicates}

The nebular chemistry is quite complex. The central theme in the kinetic theory proposed by Marcus (2004) is a generality (as shown in Eq. (1)) of the process which governs the formation of silicates from gas phase precursors in the nebula. An equilibrium is postulated between the vibrationally excited adsorbed silicon dioxide molecule and the adsorbed species $\mathrm{SiO}(\mathrm{ads})+$ $\mathrm{O}$ (ads). It was argued that reactions proceed on the surface of a growing phase where the entropic effect kinetically enhances the reaction relative to the one driven by the volume gas phase, and as mentioned earlier that the volume-based dynamical symmetry effect for observed oxygen isotope anomalies in ozone is not applicable to the gas phase precursors of CAIs. It is attempted here to understand that mechanism (Marcus 2004) of formation of early silicates in the solar system and the origin of observed mass-independent oxygen isotope effect in the light of cluster chemistry, and to the best of our knowledge this approach is the starting one. The relaxed ground state cluster geometries for $\mathrm{Si}_{n} \mathrm{O}_{m}$ were only taken into account in our recently proposed mechanism (Reber et al. 2006) on the formation of silicates through condensation of $\mathrm{SiO}$, an abundant oxygen-bearing species in space. So why start with the thermodynamically most favorable configurations?

The basic difference between the thermodynamic theory of the growth process of say detected $\mathrm{Si}_{n} \mathrm{O}_{m}$ clusters in our experiment and the one that is kinetic is that the theory assumes that each cluster after its immediate formation would occupy the energetically most favorable configuration. Whereas in a kinetic description, each cluster explores its own configurations (isomerization) along a reaction coordinate at a given total energy on its way to continued growth while existing independently (Ali 1998). Understanding further the process of chemical isomerization among various nuclear geometries of a growing cluster of a given size is an important step toward a kinetic treatment of formation of silicate grains in astrophysical objects, and this has been emphasized by Ali (1998) earlier. The 
isomerization is essentially a unimolecular reaction and the process occurs by redistribution of vibrational energy of transient quasibound cluster to motion along the reaction coordinate. The total number of isomers formed from a given transient depends on how many possible ways the energy could be redistributed between vibrational degrees of freedom of the activated complex and the translational motion in the reaction coordinate. It is true that on a typical time scale characteristic of the vibration, small clusters may not pass dynamically among several local potential energy minima of their own corresponding to isomers. Because of the long residence lifetime (Ali 1998) around a given minimum compared to the time scale of the vibrations, the cluster may get trapped in that potential minimum before it passes to the minimum of ground state geometry. What then leads a natural selection process for $\mathrm{Si}_{n} \mathrm{O}_{m}$ clusters to stabilize and become trapped in their potential minima of lowest energy geometries (Reber et al. 2006)?

In the protoplanetary accretion disk CAIs are expected to form much closer to the protosun. The temperatures in the grain forming region in astrophysical objects are much higher, they are as indicated earlier usually of the order of $\sim 1500-2000 \mathrm{~K}$. Also because of the kinetic factor (Marcus 2004), the condensation temperatures of silicates are higher than expected from equilibrium calculations. Because of the existence of such high temperatures and as the compact silicate cluster structure demands higher coordination in the interior, it is the vibrationally induced chemical isomerization process that would lead to the evolution to the lowest energy geometry without turn into excited minima corresponding to a diverse set of structural isomers. The observation of abundance distribution of $\mathrm{Si}_{n} \mathrm{O}_{m}$ clusters (Table 1) in the experiment is consistent with their calculated binding stabilities (Reber et al. 2006) of ground state structures. The model calculations (Reber et al. 2006) suggest that the most effective reaction path from gas phase $\mathrm{SiO}$ to large clusters can be constructed via formation of energetically most favorable configurations of adsorbed $\mathrm{SiO}_{2}$ clusters on the surface. These results are certainly in line with the experiment and with the theoretical prediction of Marcus (2004) that formation of adsorbed $\mathrm{SiO}_{2}$ on the surface is a highly critical step toward a mechanism of condensed phase growth of minerals in high temperature clouds.

The central question that needs to be asked is this: is the generality (Eq. (1)) of the process which governs the condensation of silicates detectable in the vibrationally induced reactive isomeric transitions in $\mathrm{Si}_{n} \mathrm{O}_{m}$ clusters?

An answer to this question would ultimately require an experiment which involves two steps: (1) The experimental technique should be capable of providing information on the kinetics of the primary act of the growth process. What initiates the formation of CAI minerals under nebular conditions? (2) Direct real-time experiments (Carley et al. 2005; Stolow et al. 2004) using ultrashort laser pulses to probe non-RRKM effect on vibrationally excited adsorbed dioxide molecules such as $\mathrm{SiO}_{2}$ on the growing cluster surface. We consider our work is a beginning toward a direction that allows us to utilise the tools of cluster science and ultrafast laser spectroscopy (Carley et al. 2005) to reveal the kinetics of the formation of silicates, and this has been ably demonstrated in a recently published study (Reber et al. 2006). The threshold zero kinetic energy (ZEKE) photodetachment spectroscopy (Carley et al. 2005; Stolow et al. 2004) of negative cluster ions is most suitable to probe vibronic structures of size-selected neutral binary, e.g. ( $\mathrm{Si}, \mathrm{O})$ clusters of less than or equal to 20 atoms. Once the spectroscopic information of clusters and their equilibrium geometries are known, femtosecond time-resolved pump-probe studies are the most logical steps to measure vibrational energy dependence of rate constants of unimolecular dissociation reactions of adsorbed activated $\mathrm{SiO}_{2}$ on the surface of clusters (Reber et al. 2006) - the reverse process of bimolecular recombination kinetics; and thus providing the relevant reaction rates in the growth of silicates in astrophysical objects.

We would like to reiterate some of the current views related to the origin of this enigmatic problem of the mass-independent oxygen isotope effect in CAIs. As it stands, the almost complete lack (Nittler 2003) of a pure ${ }^{16} \mathrm{O}$ signature in natural oxide grains has abandoned the nuclear scenario to the origin. The proposed photochemical self-shielding alternative (Clayton 2002; Lyons \& Young 2005; Yurimoto \& Kuramoto 2004) to explain this oxygen isotope anomaly is not a valid mechanism because of the lack of discrete isotopic states (Thiemens 2006; Andric et al. 2004) and there is as much cross section for CO photolysis in the spectral region $90-110 \mathrm{~nm}$. There is also no correlation (Lyons \& Young 2005; Yurimoto \& Kuramoto 2004) between photochemical abundances of oxygen isotopes and the chemical condensation pathway for the formation of silicates. Unfortunately, the majority of the meteoritics researchers consider the observed oxygen isotopes in CAIs as an anomalous effect, rather than chemical mass-independent.

By this way, our work is the first experimental demonstration (Reber et al. 2006) of intermediate formation of adsorbed $\mathrm{SiO}_{2}$ clusters on the surface in the growth of silicates, and this is an important step forward and necessary prerequisite in the investigation of kinetics of condensation in oxygen-rich molecular astronomical region where gas phase and surface reactions proceed under RRKM type conditions (Ali 1998). An inspection of the relative abundances in Table 1 shows that the oxidation of a large cluster with the chemical composition $(\mathrm{SiO})_{n}\left(\mathrm{SiO}_{2}\right)_{m}$ by free atomic oxygen in a high temperature nebular environment could possibly lead to two products. One of the products of the bimolecular collisions could be $(\mathrm{SiO})_{n-1}\left(\mathrm{SiO}_{2}\right)_{m}$ due to evaporative loss of vibrationally excited adsorbed $\mathrm{SiO}_{2}$ from the surface, the other could be $(\mathrm{SiO})_{n-1}\left(\mathrm{SiO}_{2}\right)_{m+1}$ due to the incorporation of $\mathrm{O}$ (ads) and $\mathrm{SiO}$ (ads) into the growing cluster from short lived adsorbed $\mathrm{SiO}_{2}$ on the surface. The task of ultrafast time-resolved (Carley et al. 2005; Stolow et al. 2004) studies to delineate the non-RRKM effect on the lifetimes of isotope selective adsorbed $\mathrm{SiO}_{2}^{*}$ on the surface is most formidable and a long and difficult one. Despite the fact that there is no direct test as yet of the theory (Marcus 2004) of surface analog of the microcanonical transition-states, our experimental observation and model calculations (Reber et al. 2006) on the ground state structures of intermediate silica clusters along with predictions (Marcus 2004) on the nonstatistical aspects for the vibrationally excited adsorbed dioxide molecules force us to infer that the origin of anomalous abundances of oxygen isotopes in CAIs is chemical and strictly mass-independent. The future understanding of the origin of mass-independent isotopic compositions in CAIs from the perspectives of cluster chemistry would certainly afford new insights on physicochemical conditions in the solar nebula and the kinetics (Marcus 2004) of formation of grains in situ.

\section{Genesis mission}

On a three-isotope plot (Clayton et al. 1973) the two endmembers differ primarily in their oxygen isotopic ratios ${ }^{18} \mathrm{O} /{ }^{16} \mathrm{O}$ and ${ }^{17} \mathrm{O} /{ }^{16} \mathrm{O}$. The oxygen isotopic composition of the Sun is largely uncertain. In other words the solar values of ${ }^{18} \mathrm{O} /{ }^{16} \mathrm{O}$ and ${ }^{17} \mathrm{O} /{ }^{16} \mathrm{O}$ are not exactly known, yet. This may be disclosed soon by solar wind samples of GENESIS. A major objective of the 
GENESIS mission launched in 2001 was to provide the most accurate data on the oxygen isotopic abundances of the sun. Due to the failure in soft landing of a returned space capsule, it is to be seen how successfully the samples of GENESIS recovered from the damaged capsule will provide accurate abundances. However, based on the observation of $\mathrm{CO}$ infrared rovibrational bands, new solar isotopic ratios were reported (Ayres et al. 2006): ${ }^{16} \mathrm{O} /{ }^{17} \mathrm{O}=1700 \pm 220$, and ${ }^{16} \mathrm{O} /{ }^{18} \mathrm{O}=440 \pm 6$; these ratios are significantly lower than terrestrial. A major advantage of having accurate abundances for oxygen isotopes in the sun is it offers the ability to differentiate the present chemical mechanism from other models that describe the origin of oxygen isotopic variations in planetary materials. It is expected that this major chemical constraint (Marcus 2004) combined with timeresolved kinetic experiments on the mechanism of growth of minerals and a precise knowledge of the oxygen isotopic abundances of the sun would provide us a thorough understanding of the origins of formation of our solar system.

\section{Conclusions and future directions}

By uniting the chemical aspect (Reber et al. 2006) of grain formation - the observation of oxygen-enriched nanoclusters of silicates and first principles theoretical studies on their thermochemistry, structure and properties - with the isotopic aspect of the non-RRKM effect (Marcus 2004) during the formation of $\mathrm{SiO}_{2}$ on the surface of the cluster, we show that the origin of the isotopic effects in the earliest processed oxide minerals is chemical and strictly mass-independent. A merit of the chemical mechanism is that it does not require the preexistence of hypothetical multiple isotopic reservoirs in the solar nebula, and it is very general. Since the origin of the oxygen isotopic effect in CAIs is shown to be chemical, the further understanding of the chemical dynamics of the formation of nanoclusters of silicates would place a major constraint on models (Gail 2003) of the evolution of protoplanetary accretion disks. Ultrafast, timeresolved, pump-probe studies (Carley et al. 2005) would be required to probe the surface non-RRKM effect.

One of our major problems in astrochemistry is that we have failed to identify the role of reactions between isolated molecules and the growing surfaces (clusters) at high temperatures in the innermost regions of protoplanetary disks, where most of the models emphasize just one sided view, the kinetics of low temperature ion-molecule reactions and the formation of ices on dust in the far outer disk. With the Mid-Infrared Interferometric Instrument (MIDI) installed at the Very Large Telescope Interferometer (VLTI), spatially resolved detections and compositional analyses of silicates in the inner-most two astronomical units of three protoplanetary disks have finally been reported (van Boekel et al. 2004). These astronomical observations imply that the origin of crystalline silicates in almost the entire inner disk is by vaporization and subsequent direct condensation cluster reactions in the very early active phase in the evolution of the disk. These current observations of formation of silicates at high temperatures along with meteoritic findings of mass-independent oxygen isotopes which show up in CAIs, the most refractory among minerals, argue that the process (Thiemens 2006) of oxidation of silicon via the $\mathrm{H}-\mathrm{O}$ reaction network at large distances in the disk where $T$ is low is not relevant to the origin of anomalous isotope effect. Moreover, the $\eta$-effect (Marcus 2004) decreases substantially as $T$ decreases.

The formation of transient $\mathrm{SiO}_{2}$ species on the surfaces of clusters resulted the chemically anomalous $(\mathrm{SiO})_{n}\left(\mathrm{SiO}_{2}\right)_{m}$ compositions (Table 1), a significant finding in the experiment. In conclusion we thus note that a symmetry dependent surface nonRRKM effect is the most viable explanation of the origin of oxygen isotope anomalies and would represent a major chemical process in the history (McKeegan \& Leshin 2001) of formation of our solar system nearly $4.56 \times 10^{9}$ years ago. There are many astrophysical models on the formation of our own solar system (Busemann et al. 2001). These models are primarily based on current astronomical observations of circumstellar disks around young stars, and meteoritic findings of anomalous abundances of oxygen isotopes. If the non-RRKM effect in surface reactions (Marcus 2004) is the origin for the slope-1 line in CAIs, this would not only place a major constraint on models of evolution of protoplanetary disks, but would also require many established astrophysical theories to be modified altogether.

Acknowledgements. A.A. wishes to acknowledge helpful discussions and correspondence with his colleagues - Dr. A. W. Castleman Jr., Dr. R. N. Clayton, Dr. B. D. Donn, Dr. J. Farquhar, Dr. H. J. Fraser, Dr. E. Herbst, Dr. T. Hewagama, Dr. S. N. Khanna, Dr. L. A. Leshin, Dr. R. A. Marcus, Dr. D. M. Neumark, Dr. M. H. Thiemens, and Dr. E. D. Young. The comments, suggestions and citation of the paper by Ayres et al. on solar abundances by an anonymous referee were critical in improving this manuscript. The support of NASA's Cosmochemistry and Origins of Solar systems Research and Analysis Programs is acknowledged.

\section{References}

Ali, A. 1998, Faraday Discuss., 109, 373

Allegre, C. 2001, Phil. Tran. R. Soc. Lond. A, 359, 2137

Andric, L., Bouakline, F., Grozdanov, T. P., et al. 2004, A\&A, 421, 381

Ayres, T. R., Plymate, C., \& Keller, C. U. 2006, ApJS, 165, 618

Busemann, H., Binns, W. R., Chiappini, C., et al. 2001, in Solar and Galactic Composition, ed. R. F. Wimmer-Schweingruber, American Institute of Physics, 357

Carley, R. E., Heesel, E., \& Fielding, H. H. 2005, Chem. Soc. Rev., 34, 949

Charlo, D., \& Clary, D. C. 2004, J. Chem. Phys., 120, 2700

Clayton, R. N. 2002, Nature, 415, 860

Clayton, R. N., Grossman, L., \& Mayeda, T. K. 1973, Science, 182, 485

Gail, H.-P. 2003, in Lecture Notes in Physics, Astromineralogy, vol. 609, ed. Th. Henning, 55

Gao, Y. Q., \& Marcus, R. A. 2001, Science, 293, 259

Gao, Y. Q., \& Marcus, R. A. 2002, J. Chem. Phys., 116, 137

Hathorn, B. C., \& Marcus, R. A., 1999, J. Chem. Phys., 111, 4087

Hathorn, B. C., \& Marcus, R. A. 2000, J. Chem. Phys., 113, 9497

Lyons, J. R., \& Young, E. D. 2005, Nature, 435, 317

Marcus, R. A. 2004, J. Chem. Phys., 121, 8201

McKeegan, K. D., \& Leshin, L. A. 2001, in Reviews in Mineralogy and Geochemistry, ed. J. W. Valley, \& D. R. Cole, 43, 279

Nittler, L. R. 2003, Earth Planet. Sci. Lett., 209, 259

Reber, A. C., Clayborne, P. A., Reveles, J. U., et al. 2006, Nano Lett., 6, 1190

Stolow, A., Bragg, A. E., \& Neumark, D. M. 2004, Chem. Rev., 104, 1719

Thiemens, M. H. 1996, in Chondrules and the Protoplanetary Disk, ed. R. H.

Hewins, R. H. Jones, \& E. R. D. Scott (Cambridge: Cambridge University Press), 107

Thiemens, M. H. 2006, Annu. Rev. Earth Planet. Sci., 34, 217

Urey, H. C. 1947, J. Chem. Soc. London, 562

van Boekel, R., Min, M., Leinert, Ch., et al. 2004, Nature, 432, 479

Weston, R. E., Jr. 1999, Chem. Rev., 99, 2115

Yurimoto, H., \& Kuramoto, K. 2004, Science, 305, 1763 